\title{
Safety monitoring of ROTAVAC vaccine and etiological investigation of intussusception in India: study protocol
}

Samarasimha Reddy ${ }^{1}$, Nayana P. Nair ${ }^{1}$, Sidhartha Giri ${ }^{1}$, Venkata Raghava Mohan², Jacqueline E. Tate ${ }^{3}$, Umesh D. Parashar ${ }^{3}$, Mohan D. Gupte ${ }^{4}$, Rashmi Arora ${ }^{5}$, Gagandeep Kang ${ }^{1,5^{*}}$ (D) and Indian Intussusception Surveillance Network

\begin{abstract}
Background: ROTAVAC, an indigenous rotavirus vaccine, was introduced in the universal immunization program of India in four states in 2016 and expanded to five more states in 2017. The clinical trial on efficacy of ROTAVAC did not detect an increased risk of intussusception, but the trial was not large enough to detect a small risk. This protocol paper describes the establishment and implementation of a surveillance system to monitor the safety of rotavirus vaccine and investigate the potential infectious etiologies of intussusception.

Methods: This is a multi-centric hospital-based active surveillance being conducted at 28 hospitals in nine states of India. Data gathered from surveillance will be used to assess the risk of intussusception after ROTAVAC administration and to determine the infectious etiologies of intussusception. For safety assessment of ROTAVAC vaccine, children aged less than two years with intussusception admitted at the sentinel hospitals are enrolled into surveillance, a case report form completed, and a copy of the vaccination card obtained. The risk of intussusception following rotavirus vaccination will be assessed using a self-controlled case-series design. The investigation for potential infectious etiologies of intussusception is through a matched case-control design. Children enrolled for the safety assessment serve as cases and for each case, an age, gender and location matched control is enrolled within 30 days of case enrollment. Stool specimens are obtained from cases and controls. All forms and specimens are sent to the referral laboratory for data entry, analysis, multiplexed molecular testing, and storage.

Discussion: Anticipated public health benefits of this surveillance include the generation of information useful to national government on safety of vaccine and to make future decisions on vaccine use through risk-benefit analysis. Investigating infectious agents may help to determine the potential infectious etiologies of intussusception.
\end{abstract}

Keywords: ROTAVAC, Rotavirus vaccine, Intussusception, India, Self-controlled case series methods, Infectious etiologies

\section{Background}

Rotavirus (RV) is the commonest cause of severe gastroenteritis worldwide, accounting for 215,000 deaths annually among children under five years of age [1]. In India, based on the 2011 birth cohort, RV gastroenteritis causes an estimated 11.37 million illness episodes, 3.27 million outpatient visits and 872,000 inpatient admissions each year resulting in direct costs of USD 172.8 million each year [2]. RV

\footnotetext{
* Correspondence: gkang@cmcvellore.ac.in

${ }^{1}$ The Wellcome Trust Research Laboratory, Division of Gastrointestinal Sciences, Christian Medical College, Vellore, Tamil Nadu, India

${ }^{5}$ Translational Health Science and Technology Institute, Faridabad, India Full list of author information is available at the end of the article
}

caused approximately $39 \%$ of gastroenteritis hospitalizations and 78,000 deaths among Indian children under five years of age [2]. The World Health Organization (WHO) recommends the introduction of rotavirus vaccines in all countries and particularly, in countries with high child mortality due to gastroenteritis [3]. ROTAVAC (Bharat Biotech), an indigenously developed monovalent, live attenuated oral rotavirus vaccine containing the $116 \mathrm{E}$ strain (G9P[11]) [4], is being introduced in the Universal Immunization Program (UIP) of India in a phased manner with initial introduction in four states in 2016 and five additional states in 2017, with others states to follow $[5,6]$.

(c) The Author(s). 2018 Open Access This article is distributed under the terms of the Creative Commons Attribution 4.0 International License (http://creativecommons.org/licenses/by/4.0/), which permits unrestricted use, distribution, and reproduction in any medium, provided you give appropriate credit to the original author(s) and the source, provide a link to the Creative Commons license, and indicate if changes were made. The Creative Commons Public Domain Dedication waiver (http://creativecommons.org/publicdomain/zero/1.0/) applies to the data made available in this article, unless otherwise stated. 
A key issue for rotavirus vaccines is safety, especially with regard to intussusception, a severe but uncommon intestinal blockage [7]. An earlier rotavirus vaccine based on a different (rhesus) strain and introduced in the United States (US) in the late 1990s [8,9] was associated with an increased risk of intussusception and hence withdrawn from the US market $[10,11]$. A low-level risk of 1-6 excess cases per 100,000 vaccinated children has been identified with both monovalent Rotarix (RV1, GlaxoSmithKline) and pentavalent Rotateq (RV5, Merck) vaccines in several highand middle-income countries $[12,13]$. The clinical trial on the efficacy of ROTAVAC vaccine did not detect an increased risk of intussusception among vaccinated infants, however, the trial was not large enough to detect a small risk [4]. Additionally, while the risk to benefit ratio of rotavirus vaccines are in favour of the vaccine $[8,14]$, this rare adverse effect has been highly publicized, and it is important to generate data on intussusception $[15,16]$. The WHO recommends data collection on intussusception for rotavirus vaccines using sentinel hospitals [17]. Therefore, establishing a network of health care facilities that recognize and manage cases of paediatric intussusception will help in evaluating the association between intussusception and ROTAVAC vaccination after introduction of the vaccine into the universal immunization programme (UIP) of India.

Although the etiology of intussusception in infancy and early childhood is not very clear, infections are commonly hypothesized to be associated with intussusception in this age group $[18,19]$. Certain viruses such as adenoviruses have been found at lead points in intussusception [18, 19]. Some clinical studies have reported high prevalence rates of certain viruses in stool samples from intussusception cases [20]. The evidence for the association of enteric pathogens with intussusception is inconclusive, as most of these studies on infectious etiology have failed to do a comparative analysis between pathogens found in intussusception stool samples versus those found in samples from age-matched healthy controls. Among the very few studies which have tried to evaluate multiple infectious etiologies using a case-control approach, a study conducted in Vietnam and Australia found a strong association with adenovirus, but not with other pathogens [21]. Similar data on infectious etiology of intussusception from Indian settings is currently lacking.

This protocol paper describes the methods for establishment and implementation of an intussusception surveillance system in India to monitor the safety of rotavirus vaccine following its introduction into the UIP and to investigate the potential infectious etiologies of intussusception.

\section{Methods}

\section{Objectives}

The objectives of intussusception surveillance are:

\section{Primary objective}

To assess the risk of intussusception following ROTAVAC administration using the self-controlled case-series method.

\section{Secondary objective}

To describe potential infectious etiologies of intussusception by testing for a wide range of enteric pathogens in stool samples of intussusception cases and matched controls using sensitive molecular methods.

\section{Project management}

The project is managed by the Christian Medical College (CMC), Vellore, and the Centers for Disease Control and Prevention (CDC), Atlanta, USA in collaboration with the Translational Health Science and Technology Institute (THSTI), Faridabad and the Indian Council of Medical Research (ICMR). CMC, Vellore is responsible for all administrative arrangements, while monitoring the surveillance is jointly done by CMC, THSTI and ICMR.

\section{Site selection and surveillance initiation}

The criteria for sentinel hospitals to participate in surveillance include the ability to diagnose and manage cases of intussusception (availability of pediatric surgeon, radiologist, equipment, and facilities to manage intussusception). We selected large tertiary care hospitals in states introducing the vaccine as part of the UIP, and requested their participation. A meeting was organised for site representatives of potential sentinel hospitals. Representative from each site were requested to provide details on the facilities available in their hospitals for managing intussusception cases and retrospective data on intussusception admissions among children aged less than two years for a period of one year (Additional file 1: Table S1). Sites with appropriate expertise and infrastructure to manage intussusception cases were selected to participate in the surveillance program. Active surveillance was initiated at 28 sentinel hospitals including two hospitals from states/union territories without rotavirus vaccine as part of the UIP, but which serve as referral centres admitting cases of intussuception from states with rotavirus vaccination (Table 1 ). A memorandum of understanding (MoU) was signed between each sentinel hospital and CMC, Vellore. During surveillance initiation, training sessions were conducted to the surveillance staff at each hospital, which includes one or more pediatric surgeons, radiologists, pediatricians/ community health physicians, and a field research assistant. 
Table 1 Sentinel hospitals in Intussusception surveillance

\begin{tabular}{|c|c|c|c|}
\hline S. No. & Surveillance Network Centers & Location & State \\
\hline 1 & Kurnool Medical College & Kurnool & Andhra Pradesh \\
\hline 2 & Government General Hospital & Kakinada & Andhra Pradesh \\
\hline 3 & King George Hospital & Vishakhapatnam & Andhra Pradesh \\
\hline 4 & Sri Venkateswara Medical College & Tirupati & Andhra Pradesh \\
\hline 5 & Sardar Vallabhai Patel Post Graduate Institute of Paediatrics & Cuttack & Odisha \\
\hline 6 & Kalinga Institute of Medical Sciences & Bhubaneswar & Odisha \\
\hline 7 & Institute of Medical Sciences and SUM Hospital & Bhubaneswar & Odisha \\
\hline 8 & Hi-Tech Hospital & Bhubaneswar & Odisha \\
\hline 9 & Pandit Bhagwat Dayal Sharma Post Graduate Institute of Medical Sciences & Rohtak & Haryana \\
\hline 10 & Shaheed Hasan Khan Mewati Government Medical College & Mewat & Haryana \\
\hline 11 & Post Graduate Institute of Medical Education and Research & Chandigarh & Chandigarh \\
\hline 12 & Sawai Man Singh Medical college & Jaipur & Rajasthan \\
\hline 13 & Rabindranath Tagore medical college & Udaipur & Rajasthan \\
\hline 14 & Dr. Sampurnanand Medical college & Jodhpur & Rajasthan \\
\hline 15 & Christian Medical College & Vellore & Tamil Nadu \\
\hline 16 & Government Vellore Medical college & Vellore & Tamil Nadu \\
\hline 17 & Institute of Child health & Chennai & Tamil Nadu \\
\hline 18 & Kanchi Kama Koti Child Trust hospital & Chennai & Tamil Nadu \\
\hline 19 & Government Medical College & Madurai & Tamil Nadu \\
\hline 20 & Government Medical College & Coimbatore & Tamil Nadu \\
\hline 21 & Jawaharlal Nehru Institute of Post-graduate Medical Education \& Research (JIPMER) & Puducherry & Puducherry \\
\hline 22 & Mahatma Gandhi Memorial Medical College & Indore & Madhya Pradesh \\
\hline 23 & NSCB Medical college & Jabalpur & Madhya Pradesh \\
\hline 24 & King George Medical College & Lucknow & Uttar Pradesh \\
\hline 25 & Institute of Medical Sciences, Banaras Hindu University & Varanasi & Uttar Pradesh \\
\hline 26 & BRD Medical College & Gorakhpur & Uttar Pradesh \\
\hline 27 & Baptist Christian Hospital & Tezpur & Assam \\
\hline 28 & Government Medical college & Guwahati & Assam \\
\hline
\end{tabular}

\section{Setting and design}

The multi-centric evaluation for intussusception is at 28 sentinel hospitals in nine states of India for four years (Fig. 1). Based on the recent figures, the sentinel hospitals should be able to admit 40-50 cases per state per year (Additional file 1: Table S1). For the primary objective, the self-controlled case-series methodology will investigate the temporal association between the transient exposure and outcome, in which the individual with the outcome of interest act as his/her own control [22]. The secondary objective of evaluating potential infectious etiologies will be performed through a matched case-control design.

\section{Subjects}

\section{Primary objective}

All children less than two years of age with intussusception presenting to sentinel hospitals are eligible for recruitment. The inclusion criteria for recruiting cases into surveillance program are; i) age less than 2 years, and ii) meeting level 1 diagnostic certainty for intussusception as per Brighton collaboration criteria. Diagnostic certainty as per level 1 Brighton collaboration criteria are the confirmation of intussusception during surgery and/or by specific radiologic findings (if reduced by pneumatic/hydrostatic/ contrast enema) or at autopsy [23].

\section{Secondary objective}

All children with intussusception enrolled into surveillance for primary objective will serve as cases. For each case identified in the surveillance, a matched control is enrolled. The criteria for matching are; i) age (within \pm one month of the case-patient's age), ii) gender and iii) location (district/region), and iv) control should have a diagnosis unrelated to any gastrointestinal illness and v) control should be from the hospital where the case was enrolled. Each control is to be enrolled within 30 days of the case-patient's enrollment. 


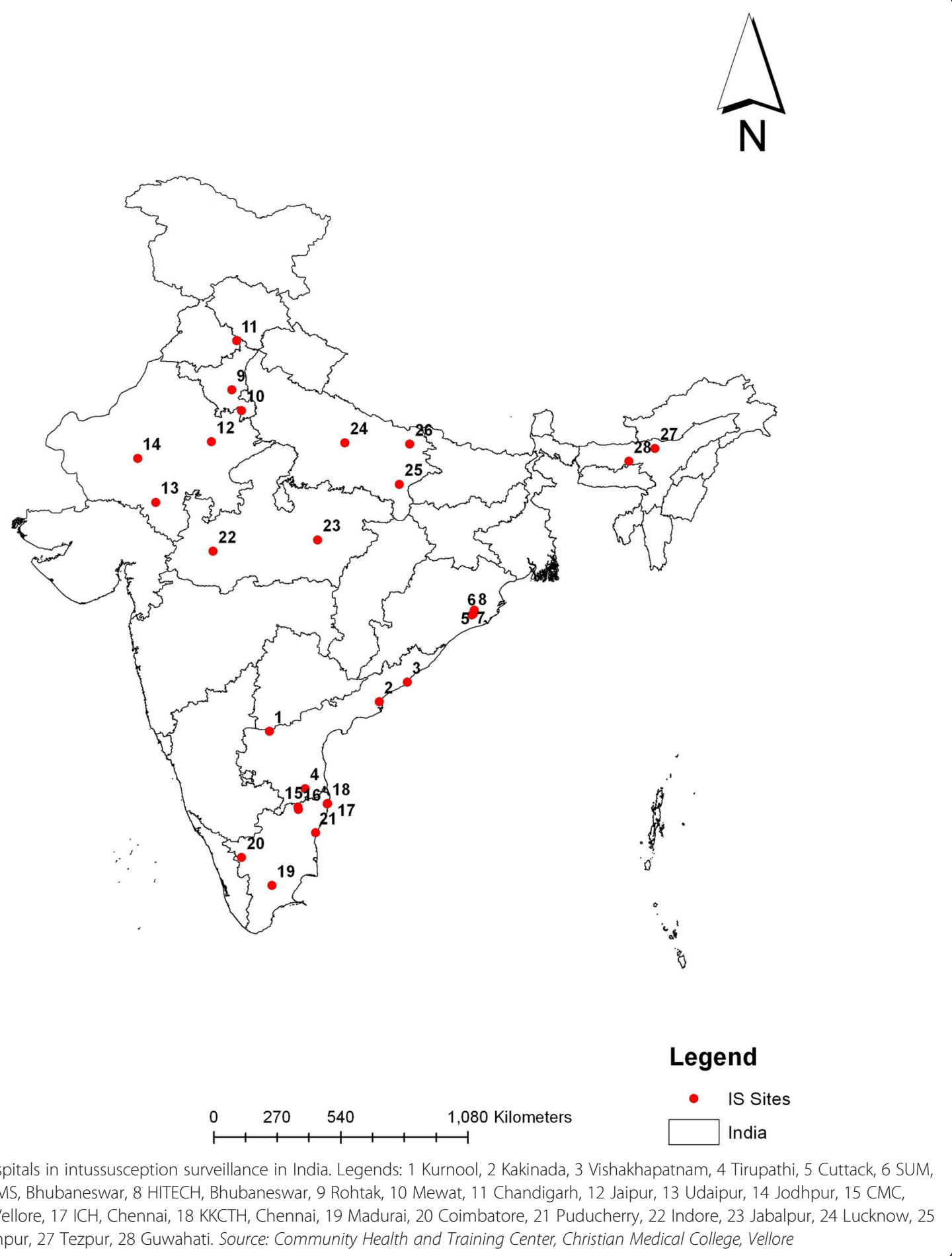

\section{Sample size}

Primary objective

Intussusception cases presenting to sentinel hospitals are to be enrolled throughout the surveillance period. To detect a relative incidence of 2 , with a 21 -day risk period after any dose, with $80 \%$ power and $5 \%$ level of significance, we require 160 intussusception cases vaccinated with ROTAVAC.

\section{Secondary objective}

To demonstrate a $10 \%$ difference in pathogen prevalence between case-patients and controls with a 
power of $80 \%$ [21], an estimated 140 case-patients and 140 controls are required.

\section{Surveillance activities Primary objective}

Surveillance staff identify intussusception cases admitted to the hospital by surveying pediatric inpatient wards, surgical theatre logs, and admission logs, in close coordination with the hospital pediatric surgeons and radiologists. On identification of a possible case, the surveillance physician ascertains the eligibility and enrols the child. The surveillance staff complete a case report form (CRF) and obtains a copy of ultrasound report along with image, hospital procedure/treatment notes and a copy of the vaccination record.

\section{Secondary objective}

A control is enrolled for each case by screening the hospital admission logs of paediatrics, pediatric surgery, and pediatric orthopaedics departments. After identifying a potential control, the surveillance physician checks all the criteria before enrollment. Once the physician ascertains the eligibility, the surveillance staff completes a CRF for the control. A stool specimen is collected from all cases and controls.

Written informed consent is obtained from parents/ legal guardians of both cases and controls prior to the enrolment. Data collection forms contain unique identifiers to permit identification of participants. At each sentinel hospital, a link between the unique identifier, name of the participant, and laboratory specimen numbers is maintained. This link between name and unique identifier and laboratory specimen will be destroyed after data collection and analysis.

\section{Specimen collection}

For the secondary objective, stool specimens are collected from both cases and controls. A bulk stool specimen $(\sim 5 \mathrm{ml})$ is obtained from each enrolled child, preferably on the day of presentation to hospital. In case of any delay, attempts are made to obtain a stool specimen within $48 \mathrm{~h}$ of hospital admission to rule out nosocomial infection. The stool specimen is collected in a sterile screw-top container labelled with a unique identification number and date of collection. At the sentinel hospitals, stool specimens are stored at $-20 \mathrm{C}$ until shipment to referral laboratory at $\mathrm{CMC}$, Vellore. Once in a month, stool specimens are sent to the referral laboratory in a vaccine carrier with frozen gel packs.

\section{Laboratory methods}

For the secondary objective, testing for the presence of multiple enteropathogens in stool samples from intussusception cases and their matched controls is by using custom made Taqman array card (TAC) assays [24]. Briefly, total nucleic acid is extracted from stool samples and tested for enteropathogen targets including enteric viruses, bacteria and parasites using arrayed singleplex real-time polymerase chain reaction (qPCR) assays. Figure 2 shows all the enteropathogen targets included on these assays for this evaluation.

\section{Data management}

Once a month, completed CRFs of all the children enrolled into surveillance (cases and controls) along with supportive documents and stool specimens are sent to the referral laboratory at $\mathrm{CMC}$, Vellore. On receipt of CRFs and stool samples, an acknowledgement e-mail communication along with any issues found with the quality and quantity of the samples is sent to the sentinel hospitals. At the central data processing centre, all CRFs are stored in a secure, locked cabinet. Trained personnel do data entry into an electronic database using structured query language. Forms with missing data are reviewed and if the program coordinator determines that it is possible to recover the data, the site investigator will attempt to obtain the missing information. Data quality for the surveillance is checked through on-going assessment of sentinel hospital performance.

\section{Monitoring surveillance}

After initiation of surveillance, sentinel sites are visited once in 3 months. At each visit, the sites are evaluated using a monitoring checklist (Additional file 2: Table S2), which recorded performance in terms of enrollment of children with intussusception, collection of ultrasound reports along with images, collection of procedure/treatment notes, enrolling the matched controls, collection of adequate stool samples and obtaining copies of the vaccination cards. Every year, a collaborators' meeting is organized to discuss the work done by each site and to enable collaborators to provide feedback to individual sites. The hospitals not meeting performance criteria are excluded from subsequent surveillance based on monitoring reports.

\section{Analysis plan \\ Primary objective}

Descriptive analyses of demographic, clinical, and treatment information will be performed for cases identified in the intussusception surveillance. The self-controlled case-series method will be used to assess the intussusception risk after ROTAVAC administration [25, 26]. The relative incidence of intussusception during the risk periods of $1-7$ days, $8-21$ days and 1-21 days post ROTAVAC vaccination for dose 1,2 and 3 will be estimated. 


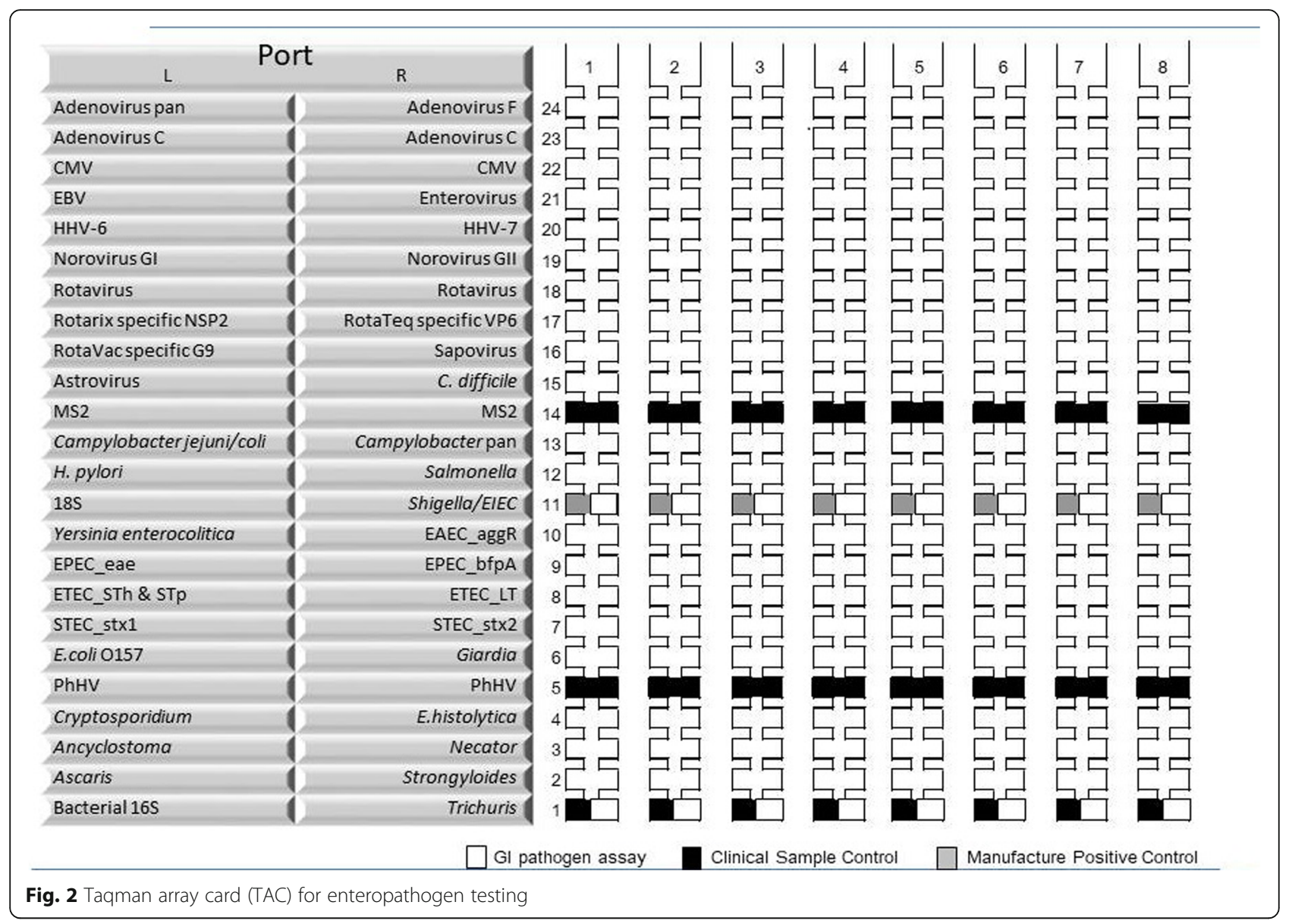

\section{Secondary objective}

Matched case-control analysis for intussusception etiologies will include comparison of demographic information, preceding clinical symptoms, and feeding patterns between case-patients and controls using the chi-square or Fisher's exact tests. Conditional logistic regression will be used to estimate adjusted population attributable fraction of intussusception due to different pathogens. Attributable cases will be calculated for each pathogen [27, 28].

\section{Discussion}

\section{Challenges in surveillance}

Initial reluctance in participation was overcome by site investigators' meetings before initiation, which helped to explain the project design, surveillance activities and significance of the project. During the surveillance, determining the vaccination status of the child and obtaining a photocopy of the vaccine card is challenging, as parents do not always carry the vaccine cards. In such cases, parents are asked to send a copy of the vaccination card by post or by email. In certain cases, the surveillance staff travels to the child's home to obtain copies. For subjects with no immunization cards available, the surveillance staff contact the auxiliary nurse midwife at health sub-centers [29] who maintain government immunization records to verify receipt of rotavirus vaccine. For children vaccinated at private hospitals, attempts are made to identify the manufacturer by contacting the health facility where the child received the rotavirus vaccine. For the etiology evaluation, enrolling controls satisfying all the matching criteria was challenging, especially at big referral hospitals having cases from two or more states. Surveillance physicians had to carefully scrutinize all the potential controls before enrolling a matched control.

Monitoring and demonstrating the safety of the vaccine in terms of intussusception after introduction of the vaccine into the routine immunization programme is important to ensure continued support and commitment for the rotavirus vaccination program. Anticipated public health benefits from this surveillance include the generation of information useful to public health officials and to national governments in making decisions through future risk-benefit analysis of the vaccine. This evaluation will also generate evidence for other low- and middle- income countries (LMIC) planning to introduce rotavirus vaccine. 
The knowledge on the etiology of intussusception is limited worldwide [30]. Evaluating the infectious agents present in stool samples from intussusception cases and matched controls may help to determine potential infectious etiologies of intussusception, and will assist in further understanding the association, if any, between rotavirus vaccination and intussusception.

\section{Additional files}

Additional file 1: Table S1. Baseline data from sentinel hospitals included in the intussusception surveillance. (DOCX 15 kb)

Additional file 2: Table S2. Monitoring checklist for sentinel hospitals in intussusception surveillance. (DOCX $26 \mathrm{~kb}$ )

\section{Abbreviations}

CDC: Centers for Disease Control and Prevention; CMC: Christian Medical College; CRF: Case report form; ICMR: Indian Council of Medical Research; LMIC: Low- and middle- income countries; MoU: Memorandum of Understanding; RV: Rotavirus; TAC: Taqman Array Card; THSTI: Translational Health Science and Technology Institute (THSTI); UIP: Universal Immunization Programme; WHO: World Health Organization

\section{Acknowledgements}

The authors are grateful for the co-operation by all participants. Members of Indian Intussusception Surveillance Network are: Sowmiya V Senthamizh, Christian Medical College, Vellore, India. Rama Prasad GS, Kurnool Medical College, Kurnool, India. Suhasini Mekala, Kurnool Medical College, Kurnool, India. Bhaskar Reddy, Rangaraya Medical College, Kakinada, India. Goru Krishna Babu, Rangaraya Medical College, Kakinada, India. Padmalatha Pamu, Andhra Medical College, Vishakhapatnam, India. Rajendra Prasad Gorthi, Andhra Medical College, Vishakhapatnam, India. Vittal Mohan, Sri Venkateswara Medical College, Tirupathi, India. B Manohar, Sri Venkateswara Medical College, Tirupathi, India. Subal Pradhan, Sardar Vallabhai Patel Post Graduate Institute of Paediatrics, Cuttack, India.

Hiranya Mohanty, Sardar Vallabhai Patel Post Graduate Institute of Paediatrics, Cuttack, India.

Mrutunjay Dash, Institute of Medical Sciences and SUM Hospital, Bhubaneswar, Odisha.

J Bikrant Kumar Prusty, Institute of Medical Sciences and SUM Hospital, Bhubaneswar, Odisha.

Nirmal Kumar Mohakud, Kalinga Institute of Medical Sciences, Bhubaneswar, Odisha.

Subrat Mohanty, Kalinga Institute of Medical Sciences, Bhubaneswar, Odisha. Rajib Ray, Hi-Tech Hospital and Medical College, Bhubaneswar, Odisha. Prasantajyoti Mohanty, Hi-Tech Hospital and Medical College, Bhubaneswar, Odisha.

Geetha Gathwala, Pandit Bhagwat Dayal Sharma Post Graduate Institute of Medical Sciences, Rohtak, India.

KN Rattan, Pandit Bhagwat Dayal Sharma Post Graduate Institute of Medical Sciences, Rohtak, India.

Suraj Chawla, Shaheed Hasan Khan Mewati Government Medical College, Mewat, India.

Yamini MS, Shaheed Hasan Khan Mewati Government Medical College, Mewat, India.

Madhu Gupta, Post Graduate Institute of Medical Education and Research, Chandigarh, India.

Surjit Singh, Post Graduate Institute of Medical Education and Research, Chandigarh, India.

RK Gupta, Sawai Man Singh Medical College, Jaipur, India.

Arun Gupta, Sawai Man Singh Medical College, Jaipur, India.

Suresh Goyal, Rabindranath Tagore Medical College, Udaipur, India. Deendayal Sharma, Rabindranath Tagore Medical College, Udaipur, India. Pramod Sharma, Dr. Sampurnanand Medical College, Jodhpur, India. Sunil Kothari, Dr. Sampurnanand Medical College, Jodhpur, India.
Gopinath, Government Vellore Medical College, Vellore, India Balasubramaniyan S, Kanchi Kama Koti Child Trust hospital, Chennai, India. Jai Durai Raj, Kanchi Kama Koti Child Trust hospital, Chennai, India. Girish Kumar CP, National Institute of Epidemiology, Chennai, India. Sridevi A Naraayan, Institute of Child health, Chennai, India. Muthu Kumar J, Institute of Child health, Chennai, India. Kulaindaivel S, Government Medical College, Madurai, India. Vaijayanthi G, Government Medical College, Madurai, India. Hemanth Kumar B, Government Medical College, Madurai, India. Raghul Maniam, Government Medical College, Coimbatore, India Rajamani Gurusamy, Government Medical College, Coimbatore, India. Kumaravel S, Jawaharlal Nehru Institute of Post-graduate Medical Education \& Research, Puducherry, India.

Ashwitha Shenoy, Jawaharlal Nehru Institute of Post-graduate Medical Education \& Research, Puducherry, India.

Brijesh Lahoti, Mahatma Gandhi Memorial Medical College, Indore, India. Sharad Thora, Mahatma Gandhi Memorial Medical College, Indore, India. Pawan Ghanghoriya, NSCB Medical college, Jabalpur, India. Vikesh Agarawal, NSCB Medical college, Jabalpur, India. Koshy George, Baptist Christian Hospital, Tezpur, India. Sam Joel, Baptist Christian Hospital, Tezpur, India. Jayanta Goswami, Government Medical college, Guwahati, India. Ashish Wakhlu, King George Medical College, Lucknow, India Vineeta Gupta, Institute of Medical Sciences, Banaras Hindu University, Varanasi, India.

SP Sharma, Institute of Medical Sciences, Banaras Hindu University, Varanasi, India.

Mahima Mithal, BRD Medical College, Gorakhpur, India.

\section{Ethics approvals and consent to participate}

The project was approved by the institutional review boards/Ethics Committees of the Christian Medical College, Kurnool Medical College, Rangaraya Medical College, SV Medical College, King George Hospital, SCB Medical College, Institute of Medical Sciences and SUM Hospital, Kalinga Institute of Medical Sciences, HiTech Medical College and Hospital, Pandit Bhagwat Dayal Sharma Post Graduate Institute of Medical Sciences, Shaheed Hasan Khan Mewati Government Medical College, Post Graduate Institute of Medical Education and Research, Savai Man Singh Medical College, Rabindranath Tagore Medical college, Dr. Sampurnanand Medical College, Madras Medical College, Coimbatore Medical College, Mahatma Gandhi Memorial Medical College, King George Medical College, BRD Medical College, Banaras Hindu University, Madurai Medical College, Kanchi Kamakoti Childs Trust Hospital, Jawaharlal Institute of Post Graduate Medical Education and Research, Emmanuel Hospital Association, Government Medical CollegeGuwahati. Written informed consent will be taken from the parents/legal guardians of participating children.

\section{Funding}

The funding for this project is from Bill and Melinda Gates Foundation to the Translational Health Science and Technology Institute (OPP1165083) and the CDC Foundation, Atlanta with the Christian Medical College (CMC), Vellore as sub-awardee.

\section{Availability of data and materials}

The anonymised datasets generated will be shared on request.

\section{Disclosure}

The findings and conclusions in this report are those of the authors and do not necessarily represent the official position of the US Centers for Disease Control and Prevention.

\section{Authors' contributions}

GK, JET, UDP and VRM conceived the idea and designed the evaluation. SR, NPN, SG, MDG, RA and the Indian intussusception surveillance network are involved in implementing the day-to-day activities related to the project. The manuscript was drafted by SR. All authors have read and approved the final manuscript.

Consent for publication

Not applicable 


\section{Competing interests}

Venkata Raghava Mohan is an Associate Editor for BMC Public Health. The other authors declare that they have no competing interests.

\section{Publisher's Note}

Springer Nature remains neutral with regard to jurisdictional claims in published maps and institutional affiliations.

\section{Author details}

${ }^{1}$ The Wellcome Trust Research Laboratory, Division of Gastrointestinal Sciences, Christian Medical College, Vellore, Tamil Nadu, India. ${ }^{2}$ Department of Community Health, Christian Medical College, Vellore, Tamil Nadu, India. ${ }^{3}$ Centers for Disease Control and Prevention, Atlanta, GA, USA. Indian Council of Medical Research, New Delhi, India. ${ }^{5}$ Translational Health Science and Technology Institute, Faridabad, India.

Received: 17 June 2018 Accepted: 5 July 2018

Published online: 20 July 2018

\section{References}

1. Tate JE, Burton AH, Boschi-Pinto C, Parashar UD, Agocs M, Serhan F, et al. Global, regional, and National Estimates of rotavirus mortality in children $<5$ years of age, 2000-2013. Clin Infect Dis. 2016;62:S96-105.

2. John J, Sarkar R, Muliyil J, Bhandari N, Bhan MK, Kang G. Rotavirus gastroenteritis in India, 2011-2013: Revised estimates of disease burden and potential impact of vaccines. Vaccine. 2014;32(Supplement 1):A5-9.

3. WHO. Rotavirus vaccines. WHO position paper - January 2013. Wkly Epidemiol Rec. 2013;88:49-64

4. Bhandari N, Rongsen-Chandola T, Bavdekar A, John J, Antony K, Taneja S, et al. Efficacy of a monovalent human-bovine (116E) rotavirus vaccine in Indian infants: a randomised, double-blind, placebo-controlled trial. Lancet. 2014:383:2136-43

5. Shri JP Nadda launches Rotavirus vaccine as part of Universal Immunization Programme; terms it a "historic moment" [Internet]. Available from: http:// pib.nic.in/newsite/PrintRelease.aspx?relid=138342 [cited 2018 Jun 17].

6. Shri JP Nadda launches expansion of Rotavirus vaccine under Universal Immunization Programme [Internet]. Available from: http://pib.nic.in/ newsite/PrintRelease.aspx?relid=158549 [cited 2018 Jun 17].

7. Murphy TV, Gargiullo PM, Massoudi MS, Nelson DB, Jumaan AO, Okoro CA, et al. Intussusception among infants given an oral rotavirus vaccine. $N$ Engl J Med. 2001;344:564-72.

8. Advisory Committee on Immunization Practices (ACIP). Rotavirus vaccine for the prevention of rotavirus gastroenteritis among children. Recommendations of the Advisory Committee on Immunization Practices (ACIP). MMWR Recomm Rep Morb Mortal Wkly Rep Recomm Rep. 1999:48:1-20.

9. Diseases $C$ on I. Prevention of rotavirus disease: guidelines for use of rotavirus vaccine. Pediatrics. 1998;102:1483-91.

10. Centers for Disease Control and Prevention (CDC). Withdrawal of rotavirus vaccine recommendation. MMWR Morb Mortal Wkly Rep. 1999:48:1007.

11. Delage G. Rotavirus vaccine withdrawal in the United States; the role of postmarketing surveillance. Can J Infect Dis. 2000;11:10-2.

12. Parashar UD, Cortese MM, Payne DC, Lopman B, Yen C, Tate JE. Value of post-licensure data on benefits and risks of vaccination to inform vaccine policy: the example of rotavirus vaccines. Am J Prev Med. 2015;49:S377-82.

13. Yen C, Healy K, Tate JE, Parashar UD, Bines J, Neuzil K, et al. Rotavirus vaccination and intussusception - science, surveillance, and safety: a review of evidence and recommendations for future research priorities in low and middle income countries. Hum Vaccines Immunother. 2016:12:2580-9.

14. Tate JE, Steele AD, Bines JE, Zuber PLF, Parashar UD. Research priorities regarding rotavirus vaccine and intussusception: a meeting summary. Vaccine. 2012;30:A179-84.

15. Yih WK, Lieu TA, Kulldorff M, Martin D, McMahill-Walraven CN, Platt R, et al. Intussusception risk after rotavirus vaccination in U.S. infants. N Engl J Med. 2014;370:503-12.

16. Weintraub ES, Baggs J, Duffy J, Vellozzi C, Belongia EA, Irving S, et al. Risk of intussusception after monovalent rotavirus vaccination. N Engl J Med. 2014; 370:513-9.

17. Julie Bines. WHO Post-marketing surveillance of rotavirus vaccine safety [Internet]. WHO. Available from: http://www.who.int/immunization/ documents/WHO_IVB_09.01/en/ [cited 2018 Jun 17].
18. Montgomery EA, Popek EJ. Intussusception, adenovirus, and children: a brief reaffirmation. Hum Pathol. 1994:25:169-74

19. Porter HJ, Padfield CJ, Peres LC, Hirschowitz L, Berry PJ. Adenovirus and intranuclear inclusions in appendices in intussusception. J Clin Pathol. 1993; 46:154-8.

20. Okimoto S, Hyodo S, Yamamoto M, Nakamura K, Kobayashi M. Association of viral isolates from stool samples with intussusception in children. Int J Infect Dis. 2011;15:e641-5.

21. Bines JE, Liem NT, Justice FA, Son TN, Kirkwood CD, de Campo M, et al. Risk factors for intussusception in infants in Vietnam and Australia: Adenovirus implicated, but not rotavirus. J Pediatr. 2006;149:452-60. e1

22. Petersen I, Douglas I, Whitaker H. Self controlled case series methods: an alternative to standard epidemiological study designs. BMJ. 2016;354:44515.

23. Bines JE, Kohl KS, Forster J, Zanardi LR, Davis RL, Hansen J, et al. Acute intussusception in infants and children as an adverse event following immunization: case definition and guidelines of data collection, analysis, and presentation. Vaccine. 2004;22:569-74.

24. Liu J, Gratz J, Amour C, Kibiki G, Becker S, Janaki L, et al. A laboratorydeveloped TaqMan Array card for simultaneous detection of 19 Enteropathogens. J Clin Microbiol. 2013;51:472-80.

25. Farrington $\mathrm{CP}$, Whitaker HJ, Hocine MN. Case series analysis for censored, perturbed, or curtailed post-event exposures. Biostatistics. 2009:10:3-16.

26. Whitaker HJ, Hocine MN, Farrington CP. The methodology of self-controlled case series studies. Stat Methods Med Res. 2009:18:7-26.

27. Bruzzi P, Green SB, Byar DP, Brinton LA, Schairer C. Estimating the population attributable risk for multiple risk factors using case-control data. Am J Epidemiol. 1985;122:904-14.

28. Blackwelder WC, Biswas K, Wu Y, Kotloff KL, Farag TH, Nasrin D, et al. Statistical methods in the global enteric multicenter study (GEMS). Clin Infect Dis. 2012;55:S246-53.

29. Indian Public Health Standards - Governnment of India [Internet]. Available from: http://nhm.gov.in/nhm/nrhm/guidelines/indian-public-healthstandards.html [cited 2018 May 5].

30. Meeting on Future Directions for Rotavirus Vaccine Research in Developing Countries (2000: Geneva, Switzerland \& WHO Department of Vaccines and Biologicals. Report of the meeting on future directions for rotavirus vaccine research in developing countries, Geneva, 9-11 February 2000. Available from: http://apps.who.int/iris/handle/10665/66497 [cited 2018 Jun 17].

Ready to submit your research? Choose BMC and benefit from:

- fast, convenient online submission

- thorough peer review by experienced researchers in your field

- rapid publication on acceptance

- support for research data, including large and complex data types

- gold Open Access which fosters wider collaboration and increased citations

- maximum visibility for your research: over $100 \mathrm{M}$ website views per year

At BMC, research is always in progress.

Learn more biomedcentral.com/submission 